\title{
Translation of in vitro findings to patients with asthma: a timely and compelling challenge
}

\author{
Pieter S. Hiemstra (10 ${ }^{1}$ and Peter J. Sterk ${ }^{2}$ \\ Affiliations: ${ }^{1}$ Dept of Pulmonology, Leiden University Medical Center, Leiden, The Netherlands. ${ }^{2}$ Dept of \\ Respiratory Medicine, Amsterdam UMC, University of Amsterdam, Amsterdam, The Netherlands. \\ Correspondence: Pieter S. Hiemstra, Leiden University Medical Center, Dept of Pulmonology, PO Box 9600, \\ Leiden, 2300 RC, The Netherlands. E-mail: P.S.HiemstraAlumc.nl
}

@ERSpublications

This editorial discusses the value of various approaches and models to study asthma, and the need for an open-minded dialogue between basic, translational and clinical scientists to discuss potential discrepancies between findings in the various models. http://bit.ly/2m5ZvJo

Cite this article as: Hiemstra PS, Sterk PJ. Translation of in vitro findings to patients with asthma: a timely and compelling challenge. Eur Respir J 2019; 54: 1901759 [https://doi.org/10.1183/13993003.01759-2019].

Our increased understanding of airway inflammation in asthma has resulted in major breakthroughs in the treatment of patients. Following the introduction of anti-inflammatory inhaled corticosteroids in the 1970s, the introduction of biologicals that selectively neutralise the activity of, for example, IgE and interleukin-5, has been a major development in targeting inflammation in subgroups of patients with severe asthma [1]. The development of these biologicals required identification of targets for therapy and proof of concept intervention studies to establish the validity of these targets. This was the result of the combined power of clinical research in patients with asthma, as well as experimental studies using in vivo and in vitro models of the disease.

The validity of such in vivo and in vitro human disease models is essential, since mice and cell cultures do not tend to develop asthma. The debate on the predictive value of animal models of asthma for understanding human asthma (referred to as "the mouse trap" $[2,3]$ ), was also spurred by increasing societal concern about the use of animals in medical research. Limitations of in vitro models are also evident, and include the selection of the right cell type(s), the influence of culture medium, matrix composition and stiffness, as well as the role of the microenvironment on the cellular response to stimuli [4]. Needless to say that results from such in vitro studies require verification in "real world" samples from patients: in vivo verification of in vitro results.

In his challenging perspective in this issue of the European Respiratory Journal [5], Carl Persson argues that findings from in vivo observations in asthma are often overlooked, because of the dominance of in vitro pathogenetic research based on cell culture, studies of isolated cell populations and molecular biology approaches. Without any doubt, the title of his perspective ("In vivo observations provide insight into roles of eosinophils and epithelial cells in asthma”) is absolutely correct. Persson [5] uses various examples to argue that multiple concepts in asthma pathogenesis have been established, without paying attention to what actually can be observed in vivo. He specifically focuses on observations in patients and studies in guinea pigs providing evidence for plasma exudation into the airways, eosinophil cytolysis and epithelial cell loss. 
PERSSON [5] argues that plasma-derived rather than epithelial-derived molecules may be essential for providing defence and repair molecules to the epithelial surface of the lung in health and asthma. The work of C. Persson and his co-workers in Lund in Sweden has indeed provided evidence that plasma exudation may be a non-injurious physiological process that provides protection of epithelial surfaces [6]. However, the epithelium also produces a range of growth factors and host defence mediators that in part differ from those present in plasma [7]. Therefore, it appears more likely that plasma- and epithelial cell-derived mediators act in concert to provide protection and repair responses, rather than the epithelium being exclusively dependent on factors derived from plasma. The ability of cultured airway epithelial cells to kill respiratory pathogens and to repair upon injury is testimony to their ability to exert these functions irrespective of the presence of plasma components, but does not provide evidence of their relative importance. It will therefore be interesting to study the composition of the mucosal lining for the relative abundance of, for example, plasma-derived antimicrobial peptides and those produced by the airway epithelium. This is feasible, because of the cell type specific spectrum of peptides produced [8], but may also be challenging because of the lack of high-quality reagents to adequately assess local levels of peptides and the difficulty in obtaining native mucosal secretions.

How about resolution of eosinophilic inflammation and elimination of eosinophils? Is eosinophil apoptosis indeed a major mechanism of regulated cell death that mediates resolution of inflammation in asthma? Various forms of regulated cell death have been identified in the lung (and other organs), including not only apoptosis, but also necroptosis, pyroptosis and autophagy (recently reviewed by SAULER et al. [9]). PERSSON [5] argues that there is no in vivo evidence for eosinophil apoptosis in airway tissues, whereas there is evidence for cytolysis. In vitro studies have clearly provided evidence for apoptosis of eosinophils and supported the role of this process in asthma pathogenesis [10, 11]. However, biopsy studies show variable data with regard to increased eosinophil apoptosis in asthma. Unfortunately, biopsies only provide a snapshot of a chronic process and apoptotic cells are rapidly cleared by macrophages, whereas non-phagocytosed apoptotic cells may undergo secondary necrosis. Nevertheless, the critical remarks of Persson and co-workers are supported by their detailed analysis of the various modes of eosinophil death in airway tissues [12], stressing the importance of more closely linking in vitro models to (replicated) in vivo observations.

In his perspective, Persson [5] also provides other interesting examples of apparent clashes between concepts partly based on in vitro studies and in vivo observations. In vivo studies in patients, healthy controls or in animals allow the performance of experiments using whole, live organisms, whereas in vitro experiments are performed in a controlled environment outside such an organism. Therefore, the complexity of interactions that can be studied in in vivo experiments by far exceeds that which can be analysed in vitro. As in vitro models, however, become more refined, they increasingly allow the detailed analysis of complex interaction, such as using co-cultures of various cell types to mimic the microenvironment, and the gradually less futuristic body-on-chip, achieved by coupling various organs-on-chips [13]. Nevertheless, these models still fail to capture the complexity of the whole body. Therefore, performing in vitro experiments has the inherent risk of unravelling mechanisms that are only operational in a culture dish but not in the whole organism. Alternatively, they may not be able to unravel mechanisms that are operational in the whole organism, but do not function in the simplified setting of an in vitro experiment. Discrepancies between in vivo observations and findings from in vitro studies should be the topic of an open-minded, careful dialogue among clinical, translational and basic scientists, and in our experience this helps to advance our insight into the pathogenesis of asthma and that of other (lung) diseases. This is also essential in toxicology, where there is increasing pressure to replace in vivo animal studies by in vitro or in silico studies. Acceptance of the new models by regulatory authorities is a major hurdle that has to be taken, but the endorsement of in vitro skin models in the analysis of possible adverse effects of cosmetic products and their ingredients on the skin is an example of the effect of societal pressure on scientific conventions in safety testing.

This is the time to press on! The revolution of systems biology has now increased opportunities for powerful observational studies by analysing patient samples, showing the complexity of cellular systems. Such an approach has already enabled the linking of comprehensive in vivo and in vitro data regarding epithelial function in asthma [14]. Similarly, single cell RNA sequencing has resulted in the identification of apparently novel subsets of cells and differentiation trajectories within the airway epithelium [15, 16], including from those with asthma [17]. Likewise, high-dimension cytometric analysis that allows the simultaneous assessment of 30-50 cell markers provides a better insight into the immune landscape of mucosal surfaces [18]. These methods include unbiased assessments and depend on state-of-the-art bioinformatics to provide detailed information on biological processes in health and disease. Providing strong evidence of the relevance of such findings for unravelling disease processes remains essential. This is one of the main points brought to our attention by the perspective of Carl Persson. Translational 
research should be bidirectional, with clinical and in vivo research informing basic and in vitro research and vice versa. The ERS and ERJ were founded for this purpose, were they not...?

Conflict of interest: P.S. Hiemstra reports grants from Boehringer Ingelheim and Galapagos, outside the submitted work. P.J. Sterk reports being Scientific Advisor and having a formally inconsiderable interest in the start-up company Breathomix, a start-up producing eNoses and licensing a cloud application for data analysis, during the conduct of the study.

\section{References}

Bel EH, ten Brinke A. New anti-eosinophil drugs for asthma and COPD. Chest 2017; 152: 1276-1282.

Persson CG, Erjefalt JS, Korsgren M, et al. The mouse trap. Trends Pharmacol Sci 1997; 18: 465-467.

Wenzel S, Holgate ST. The mouse trap: it still yields few answers in asthma. Am J Respir Crit Care Med 2006; 174: 1173-1176.

4 Bonniaud P, Fabre A, Frossard N, et al. Optimising experimental research in respiratory diseases: an ERS statement. Eur Respir J 2018; 51: 1702133.

5 Persson C. In vivo observations provide insight into roles of eosinophils and epithelial cells in asthma. Eur Respir J 2019; 54: 1900470

6 Persson C. Airways exudation of plasma macromolecules: innate defense, epithelial regeneration, and asthma. J Allergy Clin Immunol 2019; 143: 1271-1286.

7 Hiemstra PS, McCray PB, Jr., Bals R. The innate immune function of airway epithelial cells in inflammatory lung disease. Eur Respir J 2015; 45: 1150-1162.

8 Hiemstra PS, Amatngalim GD, van der Does AM, et al. Antimicrobial peptides and innate lung defenses: role in infectious and noninfectious lung diseases and therapeutic applications. Chest 2016; 149: 545-551.

9 Sauler M, Bazan IS, Lee PJ. Cell death in the lung: the apoptosis-necroptosis axis. Annu Rev Physiol 2019; 81: $375-402$.

10 Walsh GM. Eosinophil apoptosis and clearance in asthma. I Cell Death 2013; 6: 17-25.

11 Felton JM, Lucas CD, Rossi AG, et al. Eosinophils in the lung - modulating apoptosis and efferocytosis in airway inflammation. Front Immunol 2014; 5: 302.

12 Uller L, Andersson M, Greiff L, et al. Occurrence of apoptosis, secondary necrosis, and cytolysis in eosinophilic nasal polyps. Am J Respir Crit Care Med 2004; 170: 742-747.

13 Gkatzis $\mathrm{K}$, Taghizadeh S, Huh D, et al. Use of three-dimensional organoids and lung-on-a-chip methods to study lung development, regeneration and disease. Eur Respir J 2018; 52: 1800876.

14 Jevnikar Z, Ostling J, Ax E, et al. Epithelial IL-6 trans-signaling defines a new asthma phenotype with increased airway inflammation. J Allergy Clin Immunol 2019; 143: 577-590.

15 Montoro DT, Haber AL, Biton M, et al. A revised airway epithelial hierarchy includes CFTR-expressing ionocytes. Nature 2018; 560: 319-324.

16 Plasschaert LW, Zilionis R, Choo-Wing R, et al. A single-cell atlas of the airway epithelium reveals the CFTR-rich pulmonary ionocyte. Nature 2018; 560: 377-381.

17 Vieira Braga FA, Kar G, Berg M, et al. A cellular census of human lungs identifies novel cell states in health and in asthma. Nat Med 2019; 25: 1153-1163.

18 van Unen V, Li N, Molendijk I, et al. Mass cytometry of the human mucosal immune system identifies tissue- and disease-associated immune subsets. Immunity 2016; 44: 1227-1239. 